\title{
THE CONCEPT OF MAGNETIC MINERAL SEPARATION BY PARTICLE ROTATION
}

\author{
N.R. ALLEN* \\ Ka Pty Ltd., 14 Station Lane, Exton, Tasmania 7303, Australia
}

(Received 17 July 2001; Accepted on 23 July 2001)

\begin{abstract}
At present all magnetic separators use particle attraction as the separating criterion. Differences in magnetic susceptibility then determine whether or not two mineral particles can be magnetically separated. Some separators, such as the Magstream separator, have gone a step further and combined magnetic attraction with particle specific gravity.

Mineral particles have a second, largely ignored, magnetic property that can be used as a separating criterion, and this property is particle rotation. Particle rotation applies mainly to ferromagnetic minerals, and therefore separation by magnetic particle rotation is practical only for ferromagnetic particles or particles with ferromagnetic inclusions.

Particle rotation separation, in which particles are separated only if they can be magnetically rotated, is not a replacement for conventional magnetic attraction separation. Rotation separation is intended for applications where there are two different minerals with different magnetic anisotropy, or where compositional differences within the one mineral species lead to differing magnetic anisotropies.

Particle rotation separation has applications in diamond exploration where it allows the magnetic concentration of indicator minerals, and in mineral sands separation where it can provide high ilmenite recoveries with very low chrome content.
\end{abstract}

Keywords: Magnetic susceptibility; Rotation; Magnetic anisotropy; Magnetic separation; Indicator minerals

\section{INTRODUCTION}

Conventional separation by particle magnetic attraction is based on particle magnetic susceptibility (or particle magnetic moment), while

\footnotetext{
*E-mail: nallen@vision.net.au
} 
rotation separation is based on particle magnetic anisotropy, Magnetic anisotropy is a well-known concept in mineral physics, but it is not at all well known in mineral processing. Magnetic anisotropy describes the strength with which particle magnetism is tied in to specific directions within the particle, and can be present in paramagnetic as well as in ferromagnetic minerals. In paramagnetic minerals it is generally too weak to be of much practical use for particle separation. In general, ferromagnetic particles will rotate if they are placed in a rotating external field, while paramagnetic particles will not. A particle can be weakly magnetic (compared to magnetite) and still be ferromagnetic or partly ferromagnetic.

Various types of rotating magnetic field (RMF) separators are described by Allen [1], and of these, the concept considered here is RMF rotation separation where particles will only separate if they can be magnetically rotated. The term "rotatability" can be used to loosely describe the strength of particle rotational response to a rotating magnetic field. It is possible to more rigorously quantify this response, and to measure it for individual particles [2], but that is not the subject of this paper.

Although it is a general pattern that particle rotatability is roughly proportional to magnetic susceptibility, there are exceptions where two particles may have similar magnetic susceptibilities but quite different rotatabilities. These differences can occur within a single mineral species due to sometimes quite small chemical differences [2], or they may be between different mineral species due to differences in crystallography. Sometimes it is possible to accentuate these differences through treatments such as roasting [3].

The aim of this paper is to describe the concept of RMF rotation separation and to indicate some commercial uses.

\section{THE CONCEPT OF RMF ROTATION SEPARATION}

An RMF is generated by a rotating magnet rotor fitted with alternate north-south magnetic poles around its circumference. To a stationary mineral particle resting on a stationary surface around the magnet rotor, the field appears to be rotating around an axis parallel to the axis of the magnet rotor. The effect of a rotating field can be imagined 
with reference to Fig. 1. If the magnets in the figure move to the right, the ferromagnetic particle on the surface experiences a field that appears to rotate in an anticlockwise direction, and will roll itself to the left. The structure of suitable magnet rotors is described in more detail by Allen [1].

Figure 2 illustrates an experimental but practical RMF rotation separator. This particular separator has a rotor diameter of $380 \mathrm{~mm}$ and produces 20 field rotations for each complete rotation of the magnet rotor.

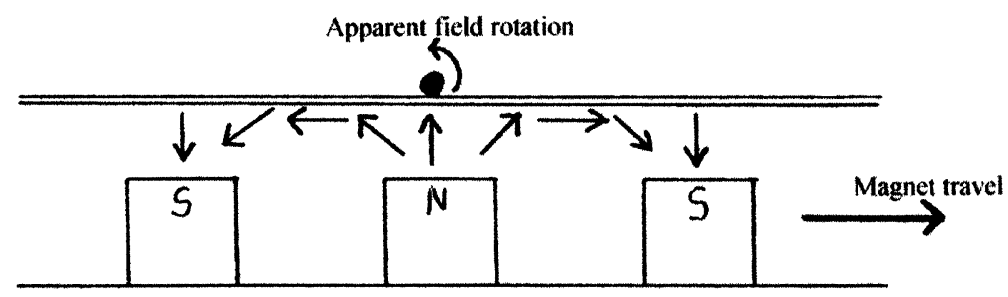

FIGURE 1 Production of a rotating magnetic field.

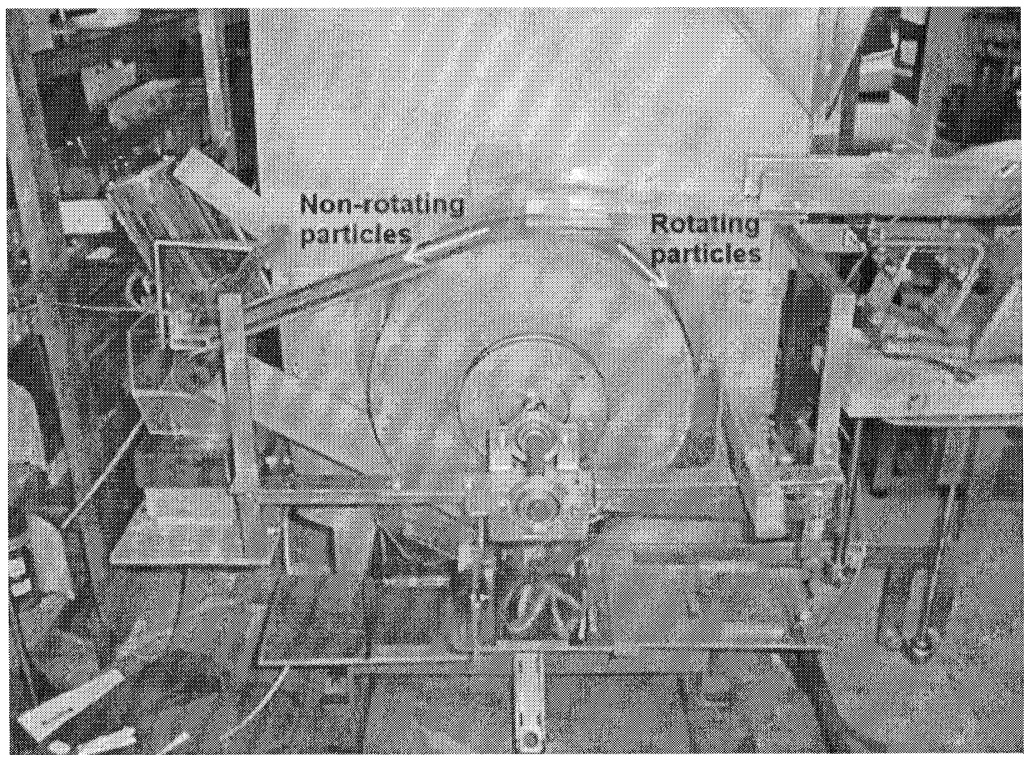

FIGURE 2 A $380 \mathrm{~mm}$ diameter experimental laboratory RMF rotation separator. 
The magnet rotor itself is inside the visible outer drum in Fig. 2, and rotates in an anticlockwise direction. This rotation produces a field that appears (to a particle on the outer drum surface) to be rotating in a clockwise direction. Therefore a ferromagnetic particle dropped onto the outer drum surface will respond by rotating in a clockwise direction in Fig. 2, and will roll itself to the right. If field rotations greater than $100 \mathrm{~Hz}$ (up to $300 \mathrm{~Hz}$ ) are used, the particle rolling speed can be very fast. The outer drum (and conveyor belt) is also rotating in an anticlockwise direction, and this carries the non-rotating particles to the left. If the belt speed is set lower than the rolling speed of the ferromagnetic particles, then there is a separation of rotating particles (roll to the right) from non-rotating particles (carried to the left).

$\mathrm{RMF}$ rotation separation is basically a separation of ferromagnetic particles from paramagnetic particles, and the concept of RMF rotation separation is therefore quite simply to roll the ferromagnetic particles in one direction while the paramagnetic particles are carried in the other.

\section{MAGNETIC MEASUREMENTS ON INDIVIDUAL PARTICLES}

The magnetic properties of ilmenite and chromite discussed below were determined from measurements of individual particle magnetic susceptibility and particle rotation characteristic. These measurements were carried out on the RMF laboratory separator, set up as shown in Fig. 3. The method is described in more detail by Allen [1,2]. Magnetic susceptibility is measured at the field required to lift the particle, and particle rotation is measured at the field required to just commence particle rotation. All measurements are made in a rotating magnetic field, and can be carried out over a range of field rotation frequencies.

\section{COMPARISON OF THE MAGNETIC PROPERTIES OF ILMENITE AND CHROMITE}

The magnetic separation of ilmenite from chrome spinel is currently receiving considerable attention within Australia, and as a result, the 


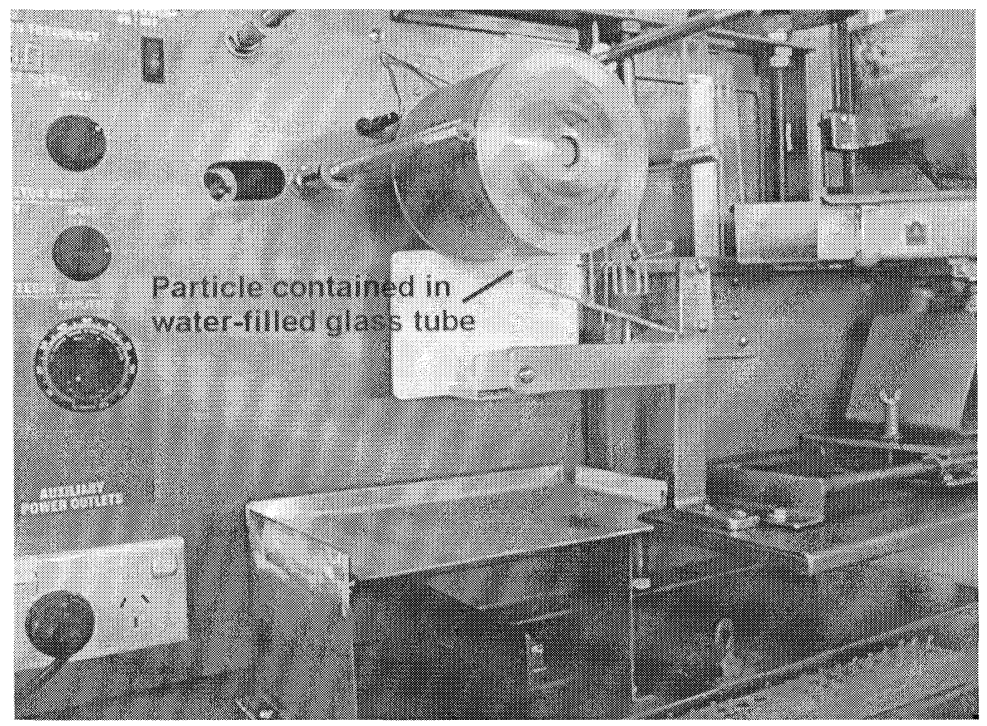

FIGURE 3 The RMF laboratory separator set up for particle magnetic measurements.

magnetic properties of these two minerals are of some interest. One of the problems encountered in the processing of ilmenite from mineral sands is the contamination of separated ilmenite with chromium. Most of this chromium is present in the form of chromite [4]. Although ilmenite has an average magnetic susceptibility higher than chromite, the average susceptibilities are quite close, and the susceptibility ranges overlap considerably.

Pure ilmenite, $\mathrm{Fe}^{2+} \mathrm{TiO}_{3}$, is paramagnetic at room temperatures and above, but most natural ilmenite contains other elements such as $\mathrm{Mg}$, $\mathrm{Mn}, \mathrm{Cr}$, and in particular $\mathrm{Fe}^{3+}$. As a result of these impurities most natural ilmenite is ferromagnetic or at least contains ferromagnetic components or inclusions. Figure 4 shows an ilmenite magnetic susceptibility frequency distribution for about 450 grains from various Australian sources.

Pure chromite is also paramagnetic at room temperatures and above, but as is the case for ilmenite, most chromite or chrome spinel is not pure and contains other elements such as $\mathrm{Fe}^{3+}$. It can be quite magnetic as a result, and this is illustrated in Fig. 4 which shows the distribution obtained from 320 chrome spinel grains. 


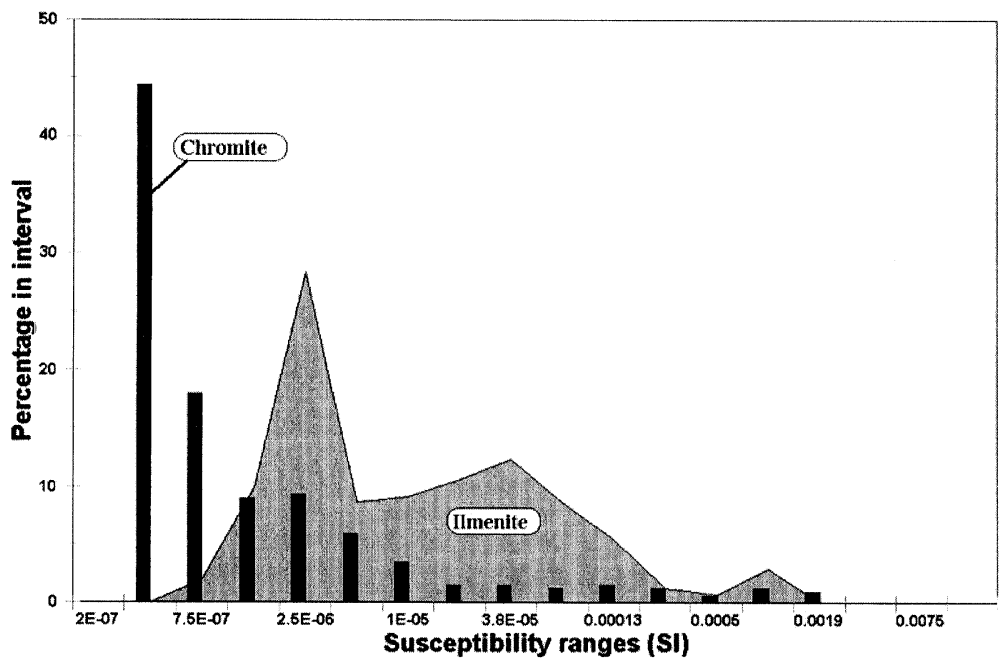

FIGURE 4 Comparison of ilmenite and chromite magnetic susceptibility ranges.

Although it is not strictly correct to talk of "susceptibility" for ferromagnetic particles that may magnetically saturate at quite low fields, the concept of susceptibility has been used here irrespective of whether the particles may be paramagnetic or ferromagnetic. Indeed, some particles may contain elements of both.

The problem faced by the conventional attraction magnetic separation of ilmenite from chromite (or chrome spinel) is readily apparent in Fig. 4. It is impossible to obtain a reasonably clean ilmenite product without discarding too much of the ilmenite. In order to obtain a good conventional attraction separation of ilmenite from chrome spinel it is first necessary to increase the magnetic susceptibility of the ilmenite, and this is the approach adopted in mineral sands separation by the roasting of the concentrate prior to magnetic separation.

Figure 5 compares the rotational characteristics of chrome spinel and ilmenite. The measured quantity here is referred to as the "rotation index" [2]. This quantity is based on the maximum attainable angle between particle magnetisation and external field, but also includes such dynamic magnetisation factors as domain wall velocity. Particle shape effects and inertia also play a part in this practical measurement. The rotation index is the measured quantity that indicates a particle's "rotatability". 


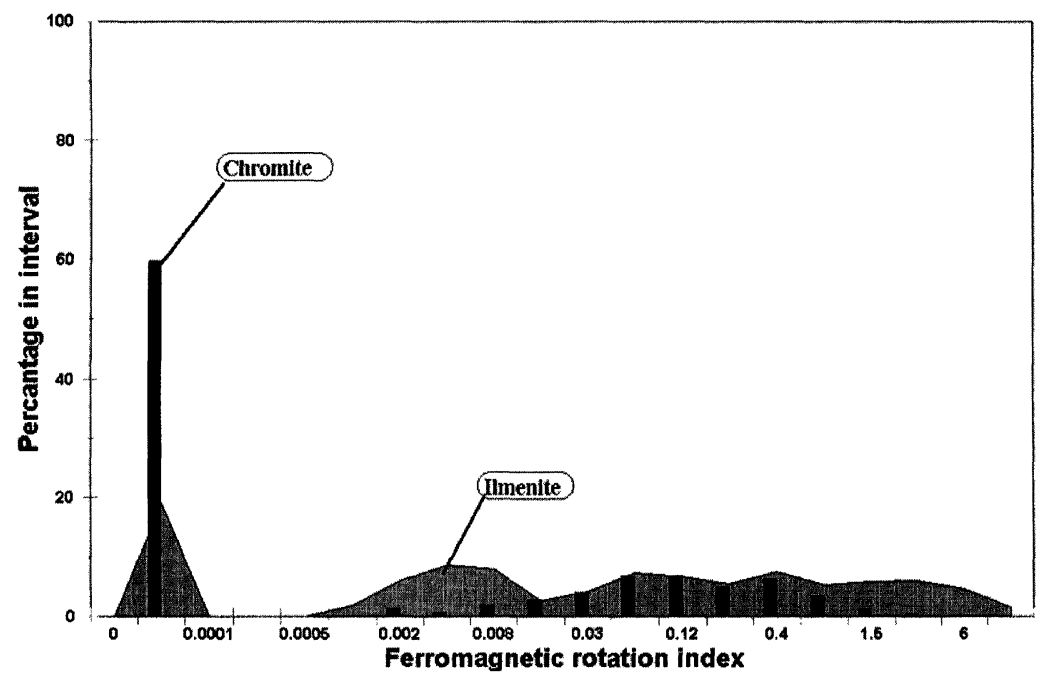

FIGURE 5 Comparison of ilmenite and chrome spinel rotation characteristics.

The particles to the right in Fig. 5 are mostly (not all) ferromagnetic, and those to the left are all paramagnetic. It is interesting to note that, while most chrome spinel has lower rotation indices than most ilmenite, there are some chrome spinel particles that rotate almost as well as the most rotatable ilmenites.

\section{THE RMF ROTATION SEPARATION OF ROASTED ILMENITE}

It has been known for some time that subjecting ilmenite to a roasting process increases its magnetic susceptibility, but that roasting does not significantly affect the chrome spinel in the same way. From Fig. 4 it can be seen that a factor of 10 increase in ilmenite susceptibility should allow a much cleaner separation between the two minerals. Fig. 5 does not seem to indicate that a similar increase in ilmenite rotatability would have much advantage (unless, at the same time, the chrome spinel rotatability is decreased). However, the magnetic properties of chrome spinel are very dependent on the source, and such magnetic 
particles are frequently not present in such numbers as shown in Fig. 5 (in this case most are closely associated with platinum ore deposits).

The work by Allen and Aral [3], that is referred to below, was carried out to examine the relationship between roasting method and particle susceptibility and rotatability, and used the separator illustrated in Fig. 2. One aim of this project was to find the best roasting method for RMF rotation separation. Five different roast conditions were tried:

Roast A Oxidising roast at $750^{\circ} \mathrm{C}$,

Roast B Oxidising roast at $750^{\circ} \mathrm{C}$ followed by a reducing roast at $850^{\circ} \mathrm{C}$,

Roast C Reducing roast at $850^{\circ} \mathrm{C}$,

Roast D Oxidising roast at $750^{\circ} \mathrm{C}$ followed by a reducing roast at $650^{\circ} \mathrm{C}$,

Roast E Reducing roast at $650^{\circ} \mathrm{C}$.

Following the roasting, an RMF rotation separation in a $0.3 \mathrm{~T}$ field was used to divide each sample into accepted and tails fractions. This procedure was carried out on five different Australian mineral sands concentrate samples. Figures 6-8 (from [3]) show the results obtained from one of the samples, NA12, that contained the highest chrome spinel content. A comparison of the RMF rotation separation with conventional magnetic attraction separation indicated an increase in ilmenite recovery by the RMF rotation separation of between $10 \%$ (for the A roasts) and $200 \%$ (for one of the E roasts). For samples that had been previously discarded as "tails", the E roast followed by RMF rotation separation performed particularly well, but conventional attraction separation was unable to accomplish worthwhile further separation irrespective of roast conditions.

A comparison of the RMF rotation separation with conventional magnetic attraction separation indicated an increase in ilmenite recovery by the RMF rotation separation of between $10 \%$ (for the A roasts) and $200 \%$ (for one of the $E$ roasts).

Figures 6-8 indicate that the best combination of high recovery and very low chrome spinel content was obtained with the low temperature reducing E roast. On sample NA12 this was in spite of the relatively high chrome spinel content of this sample. The chrome spinel content 


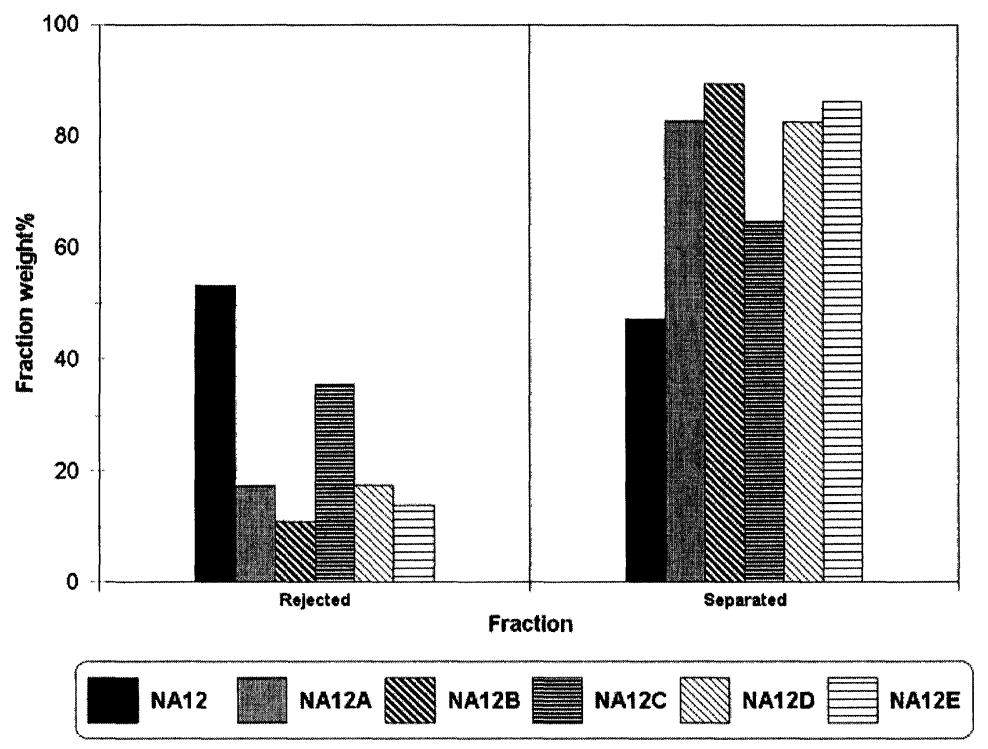

FIGURE 6 The effects of roasting on NA12 separated weight percent (from [3]).

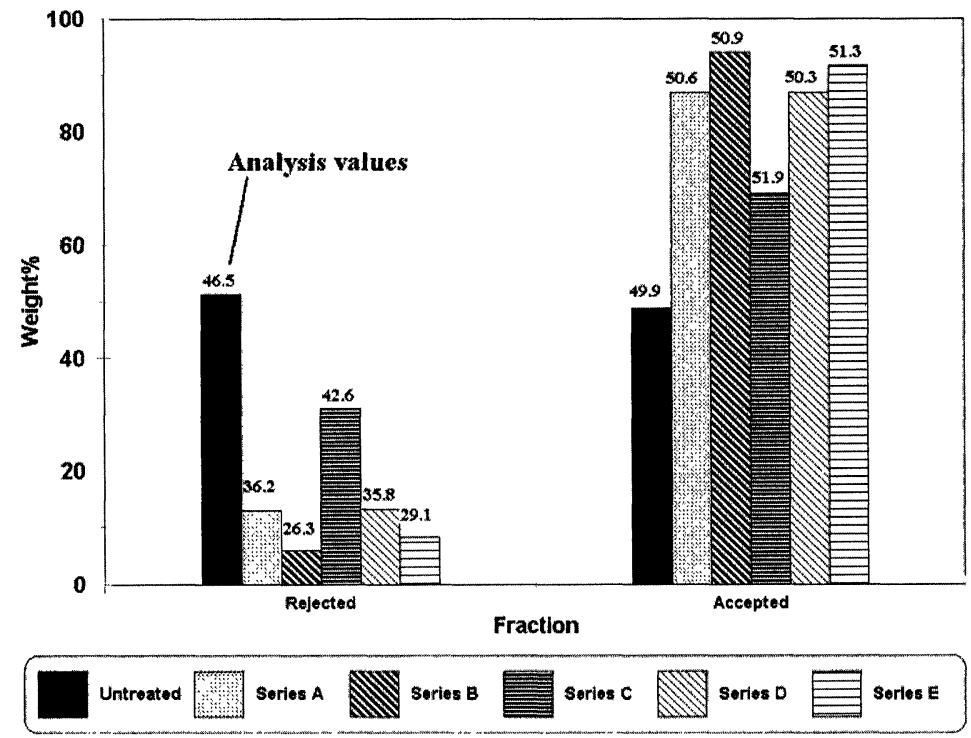

FIGURE 7 The effects of roasting on $\mathrm{NA12} \mathrm{TiO}_{2}$ distribution (from [3]). 


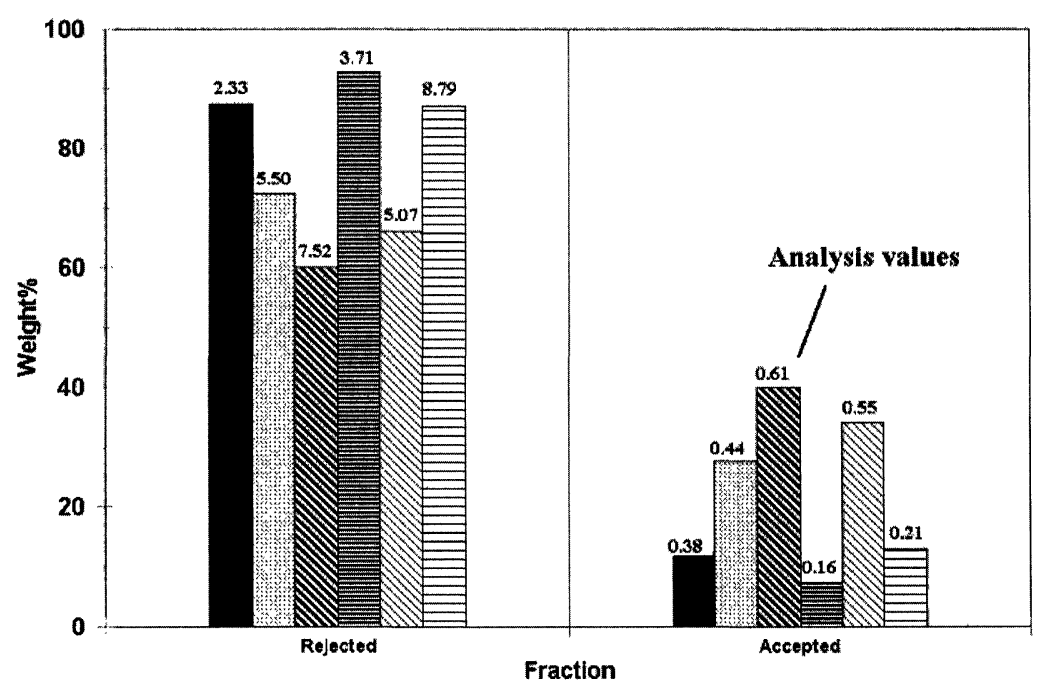

Untreated

FIGURE 8 The effects of roasting on NA12 $\mathrm{Cr}_{2} \mathrm{O}_{3}$ distribution (from [3]).

for all E roasted samples varied from 0.09 to $0.21 \%$, and was therefore below to well below commercial requirements of $0.2-0.3 \% \mathrm{Cr}_{2} \mathrm{O}_{3}$ for all samples.

The $\mathrm{E}$ roast produced some very interesting magnetic changes within the ilmenite particles, in that the magnetic susceptibility of the ilmenite was not significantly increased by the roast, but the magnetic rotatability of the particles was greatly increased. This points to a roast-induced change of only the structure of ferromagnetic components within the ilmenite, and explains why the conventional attraction separation could not accomplish reasonable recoveries on $\mathrm{E}$ roasted ilmenite. The oxidising roasts increased both susceptibility and rotatability.

The results indicate that none of the tested samples contained many of the more highly rotatable chrome spinels shown in Fig. 5. If significant numbers of these spinels had been present, RMF rotation separation (or any magnetic separation) would have been less effective in reducing the chrome spinel content. 


\section{MAGNETIC CHARACTERISTICS OF Mg AND NON-Mg ILMENITES}

One of the indicator minerals sought after in diamond exploration is picro ilmenite, a high-Mg ilmenite where many of the $\mathrm{Fe}^{2+}$ ions are replaced by $\mathrm{Mg}$. The practical laboratory problem is to find maybe only one small picro ilmenite grain in up to several kilograms of other (non-Mg) ilmenites. This is a very much easier task if the sample size can be substantially reduced, and magnetic separation is a way to accomplish the reduction.

Figure 9 shows the magnetic susceptibility range of picro ilmenites, superimposed on the "normal" ilmenite susceptibility distribution. The picro ilmenites have magnetic susceptibilities that extend across a significant proportion of the total ilmenite range. A conventional susceptibility-based magnetic separation could reduce the sample size by removing the highly magnetic ilmenites, and this is routinely done at present. However, this still leaves a large group of ilmenite particles that cannot be removed from the picro ilmenites by conventional magnetic separation.

Figure 10 compares the rotational characteristics of picro ilmenites and other ilmenites. Most picro ilmenites rotate very weakly compared

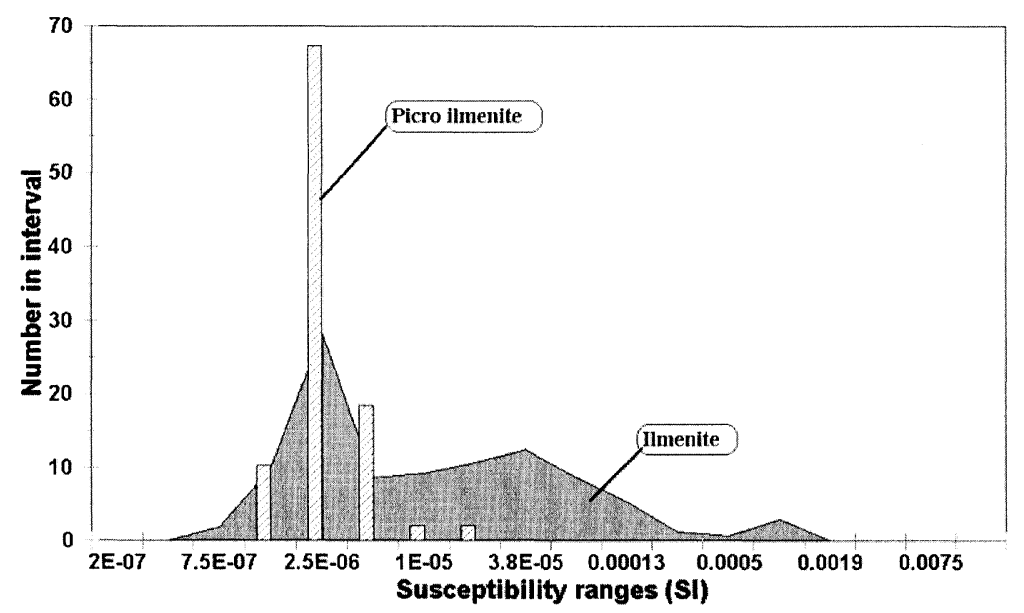

FIGURE 9 The relationship between picro ilmenite and overall ilmenite magnetic susceptibility distributions. 


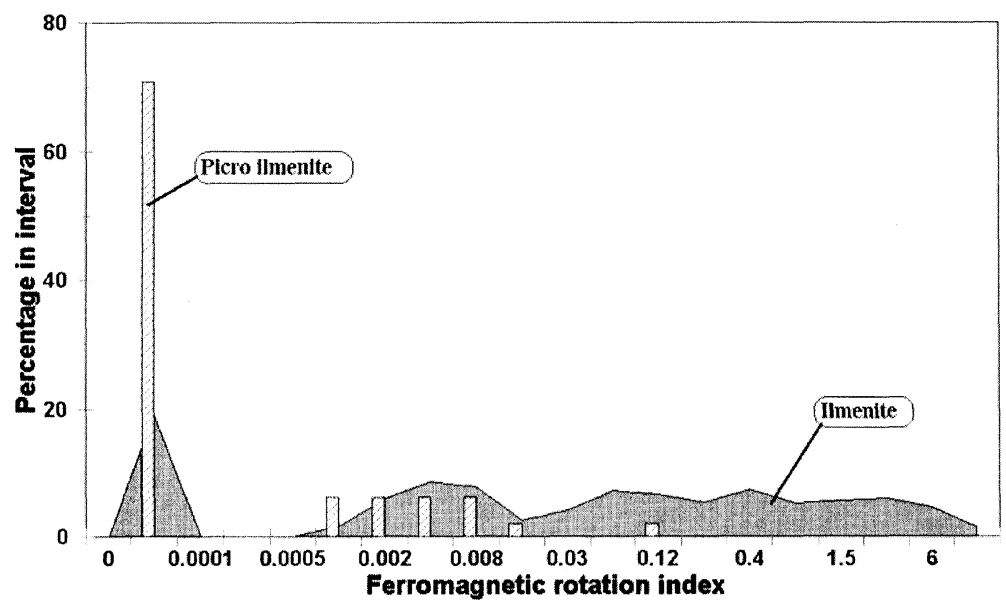

FIGURE 10 The relationship between picro ilmenite and overall ilmenite rotation index distributions.

to other ilmenites of comparable susceptibility, but there is the occasional $\mathrm{Mg}$ ilmenite (picro ilmenite?) with high $\mathrm{Fe}^{3+}$ content that does rotate strongly. An RMF rotation separation can remove a higher proportion of the sample than can be achieved with conventional attraction separation, but at the risk of losing the occasional highrotatability picro ilmenite.

\section{AN RMF DIAMOND INDICATOR SEPARATOR}

The three main indicator minerals in diamond exploration are, in order of decreasing magnetic susceptibility, picro ilmenite, Kimberlitic chromite, and pyrope garnet. The magnetic properties of picro ilmenite have been discussed above. Kimberlitic chromite does not rotate at all, and neither does pyrope garnet. Both of the latter minerals have a cubic structure and are paramagnetic and magnetically isotropic. According to various studies, the composition of Kimberlitic chromite should place it in the spin glass field, but such particles still behave as though they are paramagnetic.

The first step in a magnetic concentration of the indicator minerals is to remove a concentrated picro ilmenite fraction. The data for Fig. 9 
suggests that an attraction magnetic separation could theoretically remove about $47 \%$ of the more magnetic ilmenite for a loss of about $4 \%$ of the picro ilmenites. However, at the susceptibility values required for this, presently available laboratory separators typically give susceptibility ranges of \pm almost $100 \%$ about the average figure [1]. This means that the separation must be carried out for a much higher susceptibility in order to avoid losing too many of the picro ilmenites, and in this case would mean that only about $35 \%$ of the more magnetic ilmenite could be safely removed, for a $4-5 \%$ loss of picro ilmenites. A more accurate RMF lift separation [1], with susceptibility ranges of $\pm 50 \%$ of the average value would allow about $42 \%$ of the more magnetic ilmenite to be removed with the loss of $3-4 \%$ of the picro ilmenites.

The data for Fig. 10 suggest that an RMF rotation separation could theoretically remove about $57 \%$ of the more rotatable ilmenite for about a $4 \%$ loss of picro ilmenites. In practice this would probably reduce to around $52-54 \%$ for the same picro ilmenite loss.

The above considerations indicate that, given only the two alternatives of attraction or rotation separation, a rotation separation would produce the best sample reduction. However, if an initial attraction separation is followed by a rotation separation, the picture is not so clear. This is because some particles will have high rotatability, but low susceptibility, and others will be the other way around. In order to see what might occur in such a two-stage separation, the data for Figs. 9 and 10 have been used to model the concentration of picro ilmenites. The initial susceptibility distribution for the ilmenite and picro ilmenite is shown in Fig. 11. Figure 12 shows the effect of an accurate RMF life separation of the more magnetic particles, and Fig. 13 shows the results of subjecting the resulting low magnetism fraction to an RMF rotation separation. The effects of the separations have been expressed in terms of a susceptibility distribution irrespective of the type of separation used.

The two lowest susceptibility groups in Fig. 13 overlap with the kimberlitic chromite susceptibility range, and if it desired to place the kimberlitic chromite into its own magnetic fraction, it is necessary to use an accurate attraction separation (RMF has minimal advantage for the remaining, mostly paramagnetic, particles). The effect of using a separation is shown in Fig. 14. 


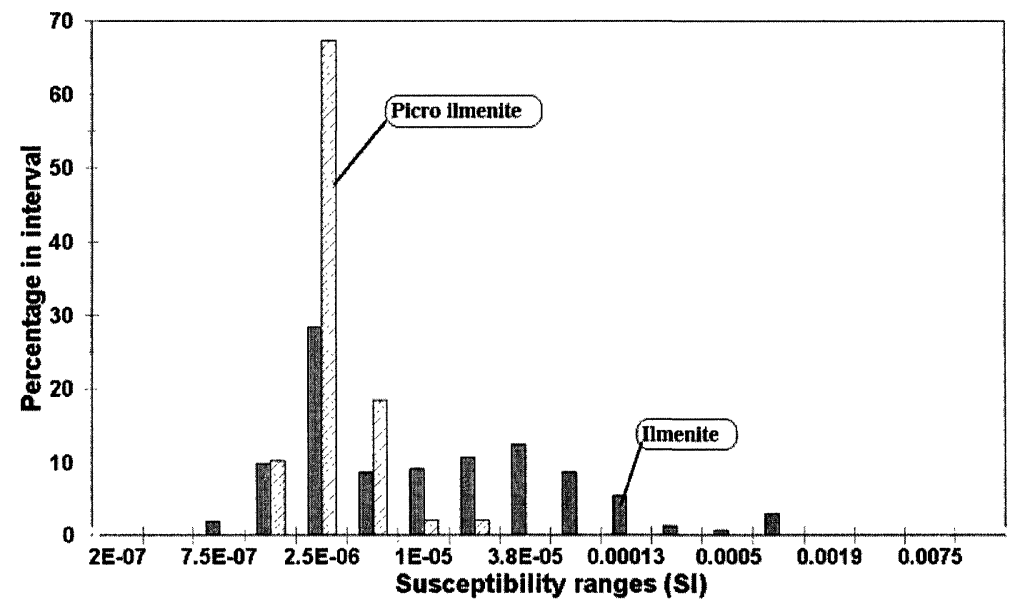

FIGURE 11 Initial susceptibility distribution of ilmenite and picro ilmenite.

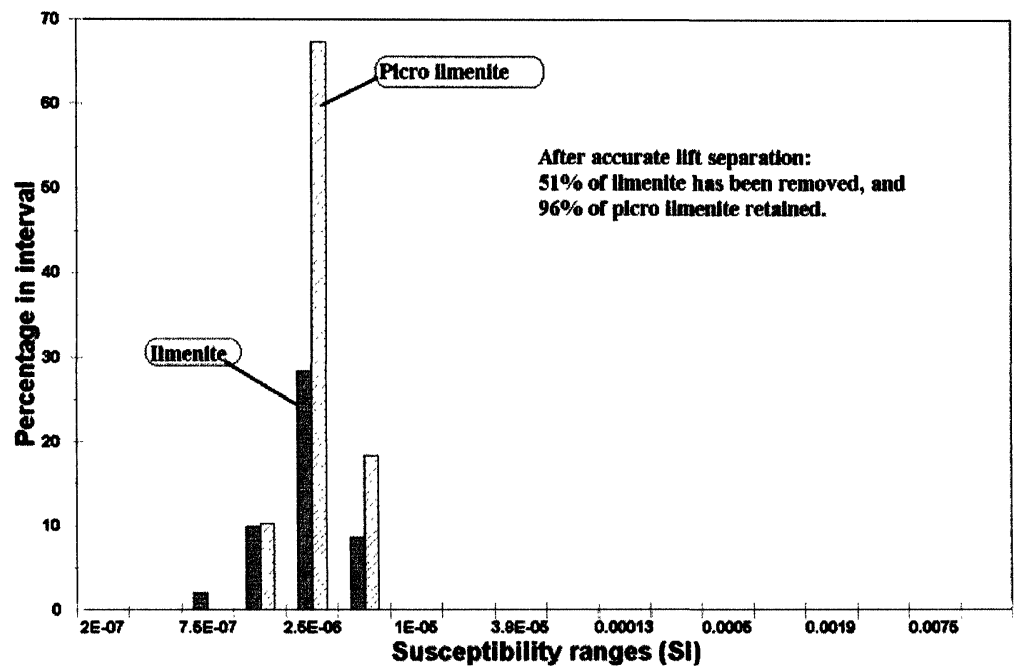

FIGURE 12 The effect of an accurate RMF life separation on ilmenite/picro ilmenite sample.

An accurate multiple-stage wet RMF separator designed for the above separation, which also provides two further stages of magnetic separation, is shown in Fig. 15. This separator has a feed capacity of only $2 \mathrm{~kg} / \mathrm{h}$ for $0.4-0.5 \mathrm{~mm}$ particles, and uses water to both dampen 


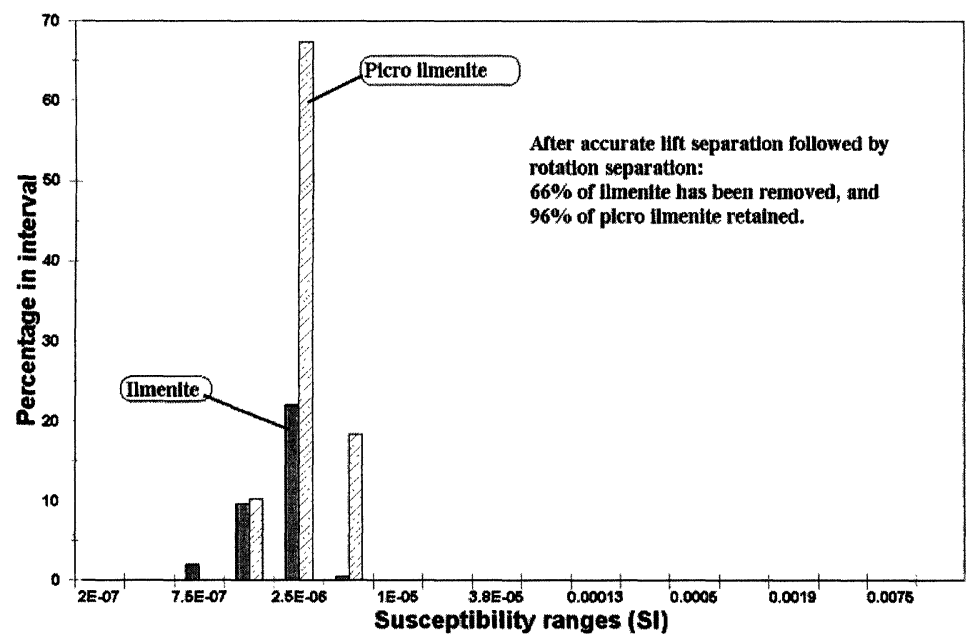

FIGURE 13 The effect of an accurate RMF rotation separation on the tails from Fig. 11 .

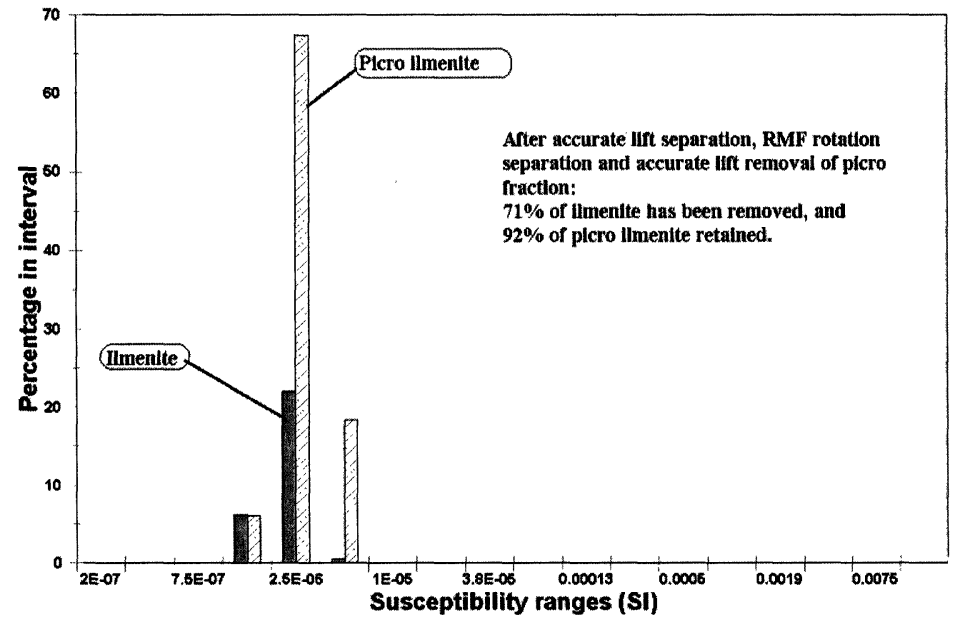

FIGURE 14 The effect of removing a Kimberlitic chromite fraction from the picro ilmenite fraction.

erratic particle motion and move particles between the different separation cells. The separator is intended for feed from which the very highly magnetic material (e.g. any magnetite) has already been removed. It has two RMF lift separation stages, followed by an RMF rotation 


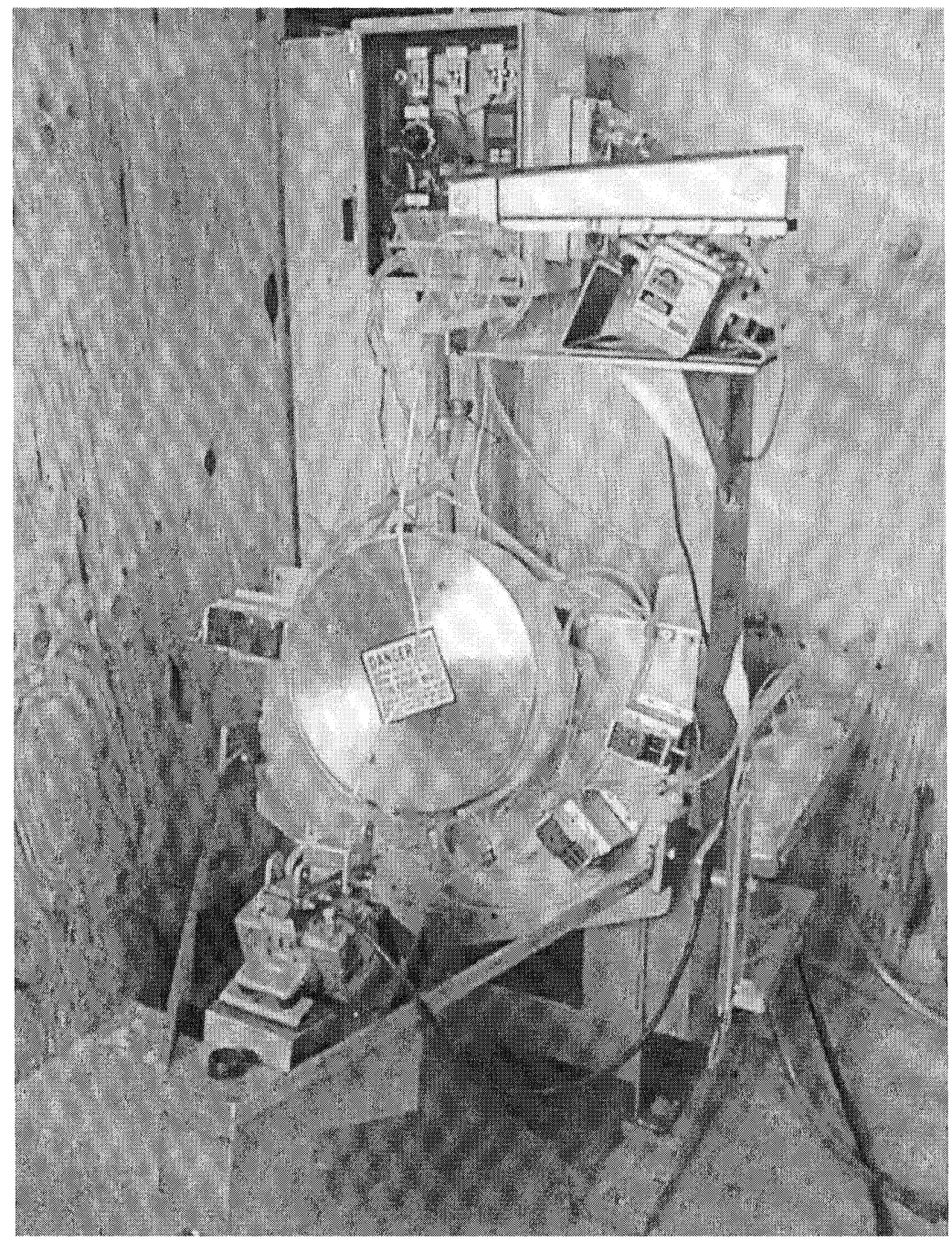

FIGURE 15 A separator for diamond indicator minerals.

separation, followed by three further stages of attraction separation (only two of them RMF).

Experience with the separator on feeds from many different sources have produced average results similar to those indicated above for the separation modelling, but there can be considerable variation between 
different source material. For some samples it is possible to reduce sample sizes by more that $95 \%$, while for other (fortunately rare) samples containing mostly paramagnetic ilmenite, the sample size reduction can be as low as $20 \%$.

An interesting possibility, in the light of the roasted ilmenite separations mentioned above, would be the development of a roast (or other) technique to change the rotatability of "normal" ilmenite while leaving the picro ilmenite relatively unaffected.

\section{DISCUSSION AND CONCLUSIONS}

There are actually three different applications discussed above:

1. RMF rotation separation of roasted ilmenite.

2. RMF separation of diamond indicator minerals

3. The modelling of multi-stage two-criteria magnetic separations based on particle magnetic measurements.

The RMF rotation separation of roasted ilmenite has opened up some interesting and previously unsuspected magnetic separation possibilities. Particle rotational properties can be changed independently of magnetic susceptibility, and rotation separation can provide high recoveries with improved purity. With the possibility now of artificially modifying either magnetic susceptibility or magnetic rotatability (or both), the application field for magnetic separation has been considerably widened.

The employment of both attraction and rotation separation in the concentration of diamond indicator minerals illustrates that the combination of both techniques into a separation sequence can produce better results than can be obtained with either technique individually. An important point here is that rotation separation is not a replacement for conventional attraction separation, it is an additional technique that produces different results.

Among particles of any given susceptibility interval, differences in chemical composition, crystallography and inclusions will produce differing rotatabilities. The results that can be achieved by the application of a combination of attraction and rotation separation are not immediately obvious from a separate knowledge of particle 
susceptibilities and rotatabilities in a sample. However, if magnetic measurements are made on representative groups of particles from each mineral component of a sample, the expected results of applying a combination of the separation methods can be modelled.

\section{References}

[1] N.R. Allen (2000). Low intensity rotating magnetic field separation, In: Proceedings MINPREX 200 International Congress of Mineral Processing and Extractive Metallurgy, pp. 303-313 (Australasian Institute of Mining and Metallurgy, Victoria, Australia).

[2] N.R. Allen (1999). Rotating magnetic separation of minerals, $\mathrm{PhD}$ thesis (unpublished), pp. 284. University of Tasmania, Hobart, Tasmania.

[3] N.R. Allen and H. Aral (2001). Application of rotating magnetic field (RMF) to the separation of roasted ilmenite. In: Proceedings International Heavy Minerals Conference 2001, pp. 193-200. (Australasian Institute of Mining and Metallurgy, Victoria, Australia).

[4] D.E. Freeman and G.J. Sparrow (1999). Processing Murray basin ilmenites. In: Extended Abstracts, Murray Basin Mineral Sands Conference, pp. 125-128 (Australasian Institute of Mining and Metallurgy, Victoria, Australia).

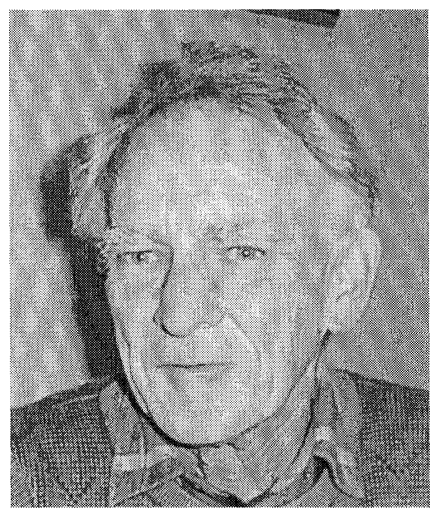

Neil Allen was born 1942. He was a Science and mathematics teacher from 1965 until early retirement in 1990. He obtained a BSc degree in 1977, a Graduate Diploma of Science with Honours in 1994, and a $\mathrm{PhD}$ in 1999 , all at the university of Tasmania. The doctoral thesis was based on the separation of minerals in a rotating magnetic field, and the company, Ka Pty Ltd, was formed during his $\mathrm{PhD}$ studies to develop the theory towards practical rotating field magnetic separators. 